\title{
EQUIVALENT NORMS ON FOCK SPACES WITH SOME APPLICATION TO EXTENDED CESARO OPERATORS
}

\author{
ZHANGJIAN HU
}

(Communicated by Richard Rochberg)

Abstract. Let $F_{\gamma}^{p}$ be the Fock space of all holomorphic functions $f$ in $\mathbf{C}^{n}$ with the Fock norm

$$
\|f\|_{p, \gamma}=\left\{\int_{\mathbf{C}^{n}}\left|f(z) e^{-\frac{\gamma|z|^{2}}{2}}\right|^{p} d A(z)\right\}^{\frac{1}{p}}<\infty,
$$

where $p, \gamma$ are positive numbers. We prove that, given any positive integer $m$, the Fock norm $\|f\|_{p, \gamma}$ is equivalent to

$$
\sum_{|\alpha| \leq m-1}\left|\partial^{\alpha} f(0)\right|+\left\{\sum_{|\alpha|=m} \int_{\mathbf{C}^{n}}\left|\partial^{\alpha} f(z)(1+|z|)^{-m} e^{-\frac{\gamma|z|^{2}}{2}}\right|^{p} d A(z)\right\}^{\frac{1}{p}} .
$$

As some application we characterize these holomorphic functions $g$ in $\mathbf{C}^{n}$ for which the induced extended Cesaro operator $T_{g}$ is bounded (or compact) from one Fock space $F_{\gamma}^{p}$ to another $F_{\gamma}^{q}$.

\section{INTRODUCTION}

Let $\mathbf{C}^{n}$ be the $n$-dimensional complex Euclidean space. For any $z=$ $\left(z_{1}, \cdots, z_{n}\right)$, we write $|z|=\sqrt{\sum_{j=1}^{n}\left|z_{j}\right|^{2}}$. Let $H\left(\mathbf{C}^{n}\right)$ be the family of all holomorphic functions in $\mathbf{C}^{n}$. For $f \in H\left(\mathbf{C}^{n}\right)$ and $\alpha=\left(\alpha_{1}, \alpha_{2}, \cdots, \alpha_{n}\right)$ a multi-index with $|\alpha|=\sum_{j=1}^{n} \alpha_{j}$, write $\partial^{\alpha} f(z)=\frac{\partial^{|\alpha|} f}{\partial^{\alpha} z}(z)$. Set $d A(z)$ to be the ordinary Lebesgue volume measure on $\mathbf{C}^{n}$. For $0<p<\infty$ and $\gamma>0$, the Lebesgue space $L_{\gamma}^{p}$ consists of all measurable functions $f$ for which

$$
\|f\|_{p, \gamma}=\left\{\int_{\mathbf{C}^{n}}\left|f(z) e^{-\frac{\gamma|z|^{2}}{2}}\right|^{p} d A(z)\right\}^{\frac{1}{p}}<\infty .
$$

We define the Fock space $F_{\gamma}^{p}=L_{\gamma}^{p} \cap H\left(\mathbf{C}^{n}\right)$. It is clear that, for $p \geq 1, L_{\gamma}^{p}$ is a Banach space under the norm $\|\cdot\|_{p, \gamma}, F_{\gamma}^{p}$ is a closed subspace of $L_{\gamma}^{p}$, and $F_{\gamma}^{2}$ is a Hilbert space. For $0<p<1, F_{\gamma}^{p}$ is an F-space under $d(f, g)=\|f-g\|_{p, \gamma}^{p}$. The Fock space has been studied in [4, 5, 6, and by some other authors.

In the setting of a Bergman space on the unit ball $B$, it is well known that $f$ is in some $p$-th Bergman space if and only if $\sum_{|\alpha|=m}\left|\partial^{\alpha} f\right|(1-|z|)^{m} \in L^{p}(B, d A)$ for some (or any) positive integer $m$. This kind of equivalence makes the Bergman space

Received by the editors April 5, 2011 and, in revised form, November 9, 2011

2010 Mathematics Subject Classification. Primary 32A37; Secondary 47B38.

Key words and phrases. Fock spaces, extended Cesaro operators.

This research was partially supported by the National Natural Science Foundation of China $(11271124,11101139)$, the Natural Science Foundation of Zhejiang Province (Y6090036, Y6100219) and the Foundation of Creative Group in Universities of Zhejiang Province (T200924). 
be connected with Bloch space and Besov space. Also, it can be applied to some other topics such as Gleason's problem on the Bergman space. See [13, 14, 16, 17. for details. Now it is natural to ask if we can characterize Fock space functions via their partial derivatives. We will prove in the next section, for $f \in H\left(\mathbf{C}^{n}\right)$, that $f \in F_{\gamma}^{p}$ if and only if $\sum_{|\alpha|=m}\left|\partial^{\alpha} f\right|(1+|z|)^{-m} \in L_{\gamma}^{p}$ for some (or any) positive integral $m$.

In [8], the extended Cesaro operator $T_{g}$ was introduced on the unit ball of $\mathbf{C}^{n}$. The behavior of $T_{g}$ on the Bergman space and the Schatten ideal property was studied respectively in [8] and [10]. It is trivial that $T_{g}$ can also be well defined on $\mathbf{C}^{n}$. Given a function $g \in H\left(\mathbf{C}^{n}\right)$, set $\Re g=\sum_{j=1}^{n} z_{j} \frac{\partial f}{\partial z_{j}}$ to be the radial derivative of $g$; see [15]. For $m=1,2, \cdots$, set $\Re^{m} f=\Re\left(\Re^{m-1} f\right)$. We give the following.

Definition. Let $g \in H\left(\mathbf{C}^{n}\right)$. The extended Cesoro operator $T_{g}$ on $H\left(\mathbf{C}^{n}\right)$ is defined as

$$
T_{g}(f)(z)=\int_{0}^{1} f(t z) \Re g(t z) \frac{d t}{t}, \quad z \in \mathbf{C}^{n} .
$$

In section 3 , with the help of our equivalent norms on $F_{\gamma}^{p}$, we will characterize those $g \in H\left(\mathbf{C}^{n}\right)$ for which the induced operator $T_{g}$ is bounded (or compact) from $F_{\gamma}^{p}$ to $F_{\gamma}^{q}$ with $0<p, q<\infty$.

In what follows, $C$ and $C_{1}, C_{2}$ will stand for positive constants whose value may change from line to line. Two quantities $A$ and $B$ are called equivalent, denoted by $A \simeq B$, if there exist $C_{1}$ and $C_{2}$ such that $C_{1} A \leq B \leq C_{2} A$.

\section{Equivalent norms on Fock spaCeS}

In this section we will give the equivalent norms on $F_{\gamma}^{p}$. To do this, we need more notation. Given $z \in \mathbf{C}^{n}$ and $\delta>0$, set the Euclidean ball $D(z, \delta)=\{w \in$ $\left.\mathbf{C}^{n}:|w-z|<\delta\right\}$ and its volume $|D(z, \delta)| \simeq \delta^{2 n}$. Set $S=\left\{z \in \mathbf{C}^{n}:|z|=1\right\}$, and set $d \sigma$ to be the rotation-invariant positive Borel measure on $S$ with $\sigma(S)=1$; see [15]. For $f \in H\left(\mathbf{C}^{n}\right)$, the $p$-th integral mean on $r S, 0<p<\infty$, is defined as $M_{p}(f, r)=\left\{\int_{S}|f(r \xi)|^{p} d \sigma(\xi)\right\}^{\frac{1}{p}}$. It is well known that $M_{p}(f, r)$ is increasing with $r \in[0, \infty)$. We write $\omega(z)=\frac{1}{1+|z|}$ for $z \in \mathbf{C}^{n}$.

Lemma 2.1. Let $\left\{k_{m}\right\}$ be a sequence of positive numbers satisfying

$$
\frac{k_{m+1}}{k_{m}} \leq c
$$

for some $c \in(0,1)$, and let $\beta>0$. Then there exist some constants $C_{1}$ and $C_{2}$ depending only on $c$ and $\beta$ such that, for all sequences $\left\{A_{m}\right\}$ of complex numbers,

$$
C_{1} \sum_{m=1}^{\infty} k_{m}\left|A_{m}\right|^{\beta} \leq \sum_{m=2}^{\infty} k_{m}\left|A_{m}-A_{m-1}\right|^{\beta}+k_{1}\left|A_{1}\right|^{\beta} \leq C_{2} \sum_{m=1}^{\infty} k_{m}\left|A_{m}\right|^{\beta} .
$$

Proof. It is easy to see that, for $a, b \in(0, \infty)$,

$$
(a+b)^{\beta} \leq \max \left(1,2^{\beta-1}\right)\left(a^{\beta}+b^{\beta}\right) .
$$

Let $\left\{A_{m}\right\}$ be a sequence of complex numbers. By (3) and (1),

$$
\sum_{m=2}^{J} k_{m}\left|A_{m}-A_{m-1}\right|^{\beta} \leq C \sum_{m=2}^{J} k_{m}\left(\left|A_{m}\right|^{\beta}+\left|A_{m-1}\right|^{\beta}\right) \leq C \sum_{m=1}^{\infty} k_{m}\left|A_{m}\right|^{\beta} .
$$


Let $J \rightarrow \infty$ to obtain

$$
\sum_{m=2}^{\infty} k_{m}\left|A_{m}-A_{m-1}\right|^{\beta}+k_{1}\left|A_{1}\right|^{\beta} \leq C \sum_{m=1}^{\infty} k_{m}\left|A_{m}\right|^{\beta} .
$$

Now we prove the reverse inequality. If $0<\beta<1$, then by (3) and (1) again,

$$
\sum_{m=1}^{J} k_{m}\left|A_{m}\right|^{\beta} \leq \sum_{k=2}^{J} k_{m}\left|A_{m}-A_{m-1}\right|^{\beta}+c \sum_{m=2}^{J} k_{m-1}\left|A_{m-1}\right|^{\beta}+k_{1}\left|A_{1}\right|^{\beta} .
$$

This implies

$$
\sum_{m=1}^{J} k_{m}\left|A_{m}\right|^{\beta} \leq C\left(\sum_{m=2}^{\infty} k_{m}\left|A_{m}-A_{m-1}\right|^{\beta}+k_{1}\left|A_{1}\right|^{\beta}\right) .
$$

If $\beta \in[1, \infty)$, by the Minkowsky inequality

$$
\begin{aligned}
& \left\{\sum_{m=2}^{J} k_{m}\left|A_{m}\right|^{\beta}\right\}^{\frac{1}{\beta}}=\left\{\sum_{m=2}^{J} k_{m}\left|\left(A_{m}-A_{m-1}\right)-A_{m-1}\right|^{\beta}\right\}^{\frac{1}{\beta}} \\
& \leq\left\{\sum_{m=2}^{J} k_{m}\left|A_{m}-A_{m-1}\right|^{\beta}\right\}^{\frac{1}{\beta}}+c^{\frac{1}{\beta}}\left\{\sum_{m=2}^{J} k_{m-1}\left|A_{m-1}\right|^{\beta}\right\}^{\frac{1}{\beta}} .
\end{aligned}
$$

With this we have (5) again. Then we have

$$
\sum_{m=1}^{\infty} k_{m}\left|A_{m}\right|^{\beta} \leq C\left\{\sum_{m=2}^{\infty} k_{m}\left|A_{m}-A_{m-1}\right|^{\beta}+k_{1}\left|A_{1}\right|^{\beta}\right\} .
$$

The estimates (2) come from (4) and (6). The proof is completed.

Lemma 2.2. Suppose $1<p<\infty, 0<s<\infty$ and $k \in \mathbf{R}$. There is some constant $C$ such that, for all locally integrable function $f$ on $[0, \infty)$,

$$
\int_{0}^{\infty}\left|\int_{0}^{r} f(t) d t\right|^{p}(1+r)^{k} e^{-s r^{2}} d r \leq C \int_{0}^{\infty}|f(r)|^{p}(1+r)^{k-p} e^{-s r^{2}} d r .
$$

Proof. We set $\varphi(r)=(1+r)^{k} e^{-s r^{2}}$ and $\psi(r)=(1+r)^{k-p} e^{-s r^{2}}$, and denote by $p^{\prime}$ the conjugate of $p$. Then by L'Hospital's rule we know that

$$
\lim _{r \rightarrow \infty} \frac{\int_{r}^{\infty} \varphi(t) d t}{r^{k-1} e^{-s r^{2}}}=\frac{1}{2 s}>0
$$

and

Therefore

$$
\lim _{r \rightarrow \infty} \frac{\int_{0}^{r} \psi(t)^{1-p^{\prime}} d t}{r^{-\frac{k-p}{p-1}-1} e^{\frac{s}{p-1} r^{2}}}=\frac{p-1}{2 s}>0 .
$$

$$
\begin{aligned}
& \lim _{r \rightarrow \infty}\left(\int_{r}^{\infty} \varphi(r) d r\right)^{\frac{1}{p}}\left(\int_{0}^{r} \psi(r)^{1-p^{\prime}} d r\right)^{\frac{1}{p^{\prime}}} \\
= & \left(\frac{1}{2 s}\right)^{\frac{1}{p}}\left(\frac{p-1}{2 s}\right)^{\frac{1}{p^{\prime}}} \lim _{r \rightarrow \infty}\left(r^{k-1} e^{-s r^{2}}\right)^{\frac{1}{p}}\left(r^{-\frac{k-p}{p-1}-1} e^{\frac{s}{p-1} r^{2}}\right)^{\frac{1}{p^{\prime}}} \\
= & \left(\frac{1}{2 s}\right)^{\frac{1}{p}}\left(\frac{p-1}{2 s}\right)^{\frac{1}{p^{\prime}}} \in(0, \infty) .
\end{aligned}
$$


Notice that $\int_{0}^{\infty} \varphi(t) d t<\infty$, and by $\psi(r)^{1-p^{\prime}} \in C[0, R]$ for all $R>0$, we have

$$
\sup _{r \in[0, \infty)}\left(\int_{r}^{\infty} \varphi(r) d r\right)^{\frac{1}{p}}\left(\int_{0}^{r} \psi(r)^{1-p^{\prime}} d r\right)^{\frac{1}{p^{\prime}}}<\infty .
$$

Apply the Riemann-Liouville Integral Theorem (see [12]) to obtain (7). The lemma is proved.

Here is the main result of the paper.

Theorem 2.1. Let $0<p, \gamma<\infty$, and let $m$ be some (or any) positive integer. Then the following three quantities are equivalent for all $f \in H\left(\mathbf{C}^{n}\right)$ :

(A) $\|f\|_{p, \gamma}$;

(B) $\sum_{|\alpha| \leq m-1}\left|\partial^{\alpha} f(0)\right|+\left\{\sum_{|\alpha|=m} \int_{\mathbf{C}^{n}}\left|\partial^{\alpha} f(z) \omega^{m}(z) e^{-\frac{\gamma|z|^{2}}{2}}\right|^{p} d A(z)\right\}^{\frac{1}{p}}$;

(C) $|f(0)|+\left\{\int_{\mathbf{C}^{n}}\left|\Re^{m} f(z) \omega^{2 m}(z) e^{-\frac{\gamma|z|^{2}}{2}}\right|^{p} d A(z)\right\}^{\frac{1}{p}}$.

In other words, we have

$$
\begin{aligned}
\|f\|_{p, \gamma} & \simeq \sum_{|\alpha| \leq m-1}\left|\partial^{\alpha} f(0)\right|+\left\{\sum_{|\alpha|=m} \int_{\mathbf{C}^{n}}\left|\partial^{\alpha} f(z) \omega^{m}(z) e^{-\frac{\gamma|z|^{2}}{2}}\right|^{p} d A(z)\right\}^{\frac{1}{p}} \\
& \simeq|f(0)|+\left\{\int_{\mathbf{C}^{n}}\left|\Re^{m} f(z) \omega^{2 m}(z) e^{-\frac{\gamma|z|^{2}}{2}}\right|^{p} d A(z)\right\}^{\frac{1}{p}} .
\end{aligned}
$$

Proof. To simplify our notation, set $t=\frac{\gamma}{2}$. First, we prove that (B) can be dominated by $(\mathrm{A})$. We claim that

$$
\frac{1}{|D(u, 2 \omega(u))|} \int_{D(u, 2 \omega(u))} e^{-t|z|^{2}} d A(z) \leq C e^{-t|u|^{2}} \text { for } u \in \mathbf{C}^{n}
$$

To see this, we can only deal with $|u| \geq 2$. For $z \in D(u, 2 \omega(u))$, then $|z|>$ $|u|-2 \omega(u)$; hence

$$
\frac{1}{|D(u, 2 \omega(u))|} \int_{D(u, 2 \omega(u))} e^{-t|z|^{2}} d A(z) \leq \sup _{|z| \geq|u|-2 \omega(u)} e^{-t|z|^{2}} \leq C e^{-t|u|^{2}} .
$$

Meanwhile, by the subharmonicity of $|f(z)|^{p}$, with the Cauchy estimate as in 11 we have some constant depending only on $p$ and $m$ such that for all $z \in \mathbf{C}^{n}$ and $|\alpha|=m$,

$$
\left|\partial^{\alpha} f(z)\right|^{p} \leq C \frac{1}{\omega(z)^{2 n+m p}} \int_{D(z, \omega(z))}|f(w)|^{p} d A(w)
$$


This gives

$$
\left|\partial^{\alpha} f(0)\right| \leq C\left(\int_{D(0,1)} \mid f(u)^{p} d A(u)\right)^{\frac{1}{p}} \leq C\|f\|_{p, \gamma}
$$

Also, it is easy to verify $|u-z|<2 \omega(u)$ if $|u-z|<\omega(z)$. Therefore, (8) and (9) yield

$$
\begin{aligned}
& \int_{\mathbf{C}^{n}}\left|\partial^{\alpha} f(z) \omega^{m}(z) e^{-t|z|^{2}}\right|^{p} d A(z) \\
\leq & C \int_{\mathbf{C}^{n}} \frac{e^{-t p|z|^{2}}}{\omega^{2 n}(z)} d A(z) \int_{\mathbf{C}^{n}}|f(u)|^{p} \chi_{D(z, \omega(z))}(u) d A(u) \\
\leq & C \int_{\mathbf{C}^{n}}\left|f(w) e^{-t|w|^{2}}\right|^{p} d A(w) .
\end{aligned}
$$

This and (10) yield $(\mathrm{B}) \leq C(\mathrm{~A})$.

For $(\mathrm{A}) \leq C(\mathrm{C})$, we suppose $n=1$ temporarily and prove

$$
\int_{\mathbf{C}}\left|f(z) \omega^{k}(z) e^{-t|z|^{2}}\right|^{p} d A(z) \leq C \int_{\mathbf{C}}\left|f^{\prime}(z) \omega^{k+1}(z) e^{-t|z|^{2}}\right|^{p} d A(z)
$$

for fixed $p>0, k \geq 0$, and for all $f \in H(\mathbf{C})$ with $f(0)=0$. If $0<p \leq 1$, Hardy's inequality implies, for $0<\rho \leq r<\infty$,

$$
M_{p}^{p}(f, r)-M_{p}^{p}(f, \rho) \leq C(r-\rho)^{p} M_{p}^{p}\left(f^{\prime}, r\right)
$$

see Lemma 3.3 in 13 . Notice that $M_{p}(f, r)$ is increasing with $r$; hence

$$
\begin{aligned}
& \int_{\mathbf{C}}\left|f(z) \omega^{k}(z) e^{-t|z|^{2}}\right|^{p} d A(z) \\
= & \sum_{s=1}^{\infty} \int_{\sqrt{s-1}}^{\sqrt{s}} M_{p}^{p}(f, r) \omega^{p k}(r) e^{-p t r^{2}} r d r \\
\leq & C \sum_{s=1}^{\infty} M_{p}^{p}(f, \sqrt{s}) \omega^{p k}(\sqrt{s}) e^{-p t s} \sqrt{s}[\sqrt{s}-\sqrt{s-1}] \\
\leq & C \sum_{s=1}^{\infty} M_{p}^{p}(f, \sqrt{s}) \omega^{p k}(\sqrt{s}) e^{-p t s} .
\end{aligned}
$$


Set $k_{s}=\omega^{p k}(\sqrt{s}) e^{-p t s}$ and $A_{s}=M_{p}^{p}(f, \sqrt{s})$ for $s=1,2, \cdots$, and $A_{0}=M_{p}^{p}(f, 0)=$ $f(0)=0$. Apply Lemma 2.1 and (12) to obtain

$$
\begin{aligned}
& \int_{\mathbf{C}}\left|f(z) \omega^{k}(z) e^{-t|z|^{2}}\right|^{p} d A(z) \\
\leq & C \sum_{s=1}^{\infty} \omega^{p k}(\sqrt{s}) e^{-p t s}\left|M_{p}^{p}(f, \sqrt{s})-M_{p}^{p}(f, \sqrt{s-1})\right| \\
\leq & C \sum_{s=1}^{\infty} \omega^{p k}(\sqrt{s}) e^{-p t s}[\sqrt{s}-\sqrt{s-1}]^{p} M_{p}^{p}\left(f^{\prime}, \sqrt{s}\right) \\
\leq & C\left\{\sum_{s=1}^{\infty} \omega^{p k}(\sqrt{s}) e^{-p t s}\left(\frac{1}{s}\right)^{\frac{p}{2}} M_{p}^{p}\left(f^{\prime}, \sqrt{s}\right)\right\} \\
\leq & C\left\{\sum_{s=1}^{\infty} \int_{\sqrt{s}}^{\sqrt{s+1}} M_{p}^{p}\left(f^{\prime}, r\right) \omega^{p(k+1)}(r) e^{-p t r^{2}} r d r\right\} \\
\leq & C \int_{1}^{\infty} M_{p}^{p}\left(f^{\prime}, r\right) \omega^{p(k+1)}(r) e^{-p t r^{2}} r d r \\
= & C \int_{\mathbf{C}}\left|f^{\prime}(z) \omega^{k+1}(z)\right|^{p} e^{-t|z|^{2}} d A(z) .
\end{aligned}
$$

If $p>1$, by Lemma 2.2 we know

$$
\begin{aligned}
\int_{0}^{\infty}\left|f(r \zeta) \omega^{k}(z) e^{-t r^{2}}\right|^{p} d r & =\int_{0}^{\infty}\left|\left(\int_{0}^{r} f^{\prime}\left(r e^{i \theta}\right) d r\right) \omega^{k}(r) e^{-t r^{2}}\right|^{p} d r \\
& \leq C \int_{0}^{\infty}\left|f^{\prime}\left(r e^{i \theta}\right) \omega^{k+1}(r) e^{-t r^{2}}\right|^{p} d r
\end{aligned}
$$

Hence, by the monotonicity of $M_{p}^{p}\left(f^{\prime}, r\right)$ we have

$$
\begin{aligned}
& \int_{\mathbf{C}}\left|f(z) \omega^{k}(z) e^{-t|z|^{2}}\right|^{p} d A(z) \\
\leq & C \int_{0}^{2 \pi}\left(\int_{0}^{\infty}\left|f\left(r e^{i \theta}\right) \omega^{k}(r) e^{-t r^{2}}\right|^{p}(1+r) d r\right) d \theta \\
\leq & C \int_{0}^{2 \pi}\left(\int_{0}^{\infty}\left|f^{\prime}\left(r e^{i \theta}\right) \omega^{k+1}(r) e^{-t r^{2}}\right|^{p}(1+r) d r\right) d \theta \\
= & C \int_{0}^{\infty}\left(\int_{0}^{2 \pi}\left|f^{\prime}\left(r e^{i \theta}\right) \omega^{k+1}(r) e^{-t r^{2}}\right|^{p}(1+r) d \theta\right) d r \\
= & C \int_{0}^{\infty} M_{p}^{p}\left(f^{\prime}, r\right)\left(\omega^{k+1}(r) e^{-t r^{2}}\right)^{p}(1+r) d r \\
= & C\left\{\int_{0}^{1}+\int_{1}^{\infty}\right\} M_{p}^{p}\left(f^{\prime}, r\right)\left(\omega^{k+1}(r) e^{-t r^{2}}\right)^{p}(1+r) d r \\
\leq & C\left\{M_{p}^{p}\left(f^{\prime}, 1\right)+\int_{1}^{\infty} M_{p}^{p}\left(f^{\prime}, r\right)\left(\omega^{k+1}(r) e^{-t r^{2}}\right)^{p} r d r\right\} \\
\leq & C\left\{\int_{1}^{2}+\int_{1}^{\infty}\right\} M_{p}^{p}\left(f^{\prime}, r\right)\left(\omega^{k+1}(r) e^{-t r^{2}}\right)^{p} r d r \\
\leq & C \int_{\mathbf{C}}\left|f^{\prime}(z) \omega^{k+1}(z) e^{-t|z|^{2}}\right|^{p} d A(z) .
\end{aligned}
$$


From (13) and (14), (11) follows. Now we go back to a higher dimension. Let $f \in$ $H\left(\mathbf{C}^{n}\right)$ with $f(0)=0$. For each $\zeta \in S$ set the slice function $f_{\zeta}(\cdot)=f(\cdot \zeta) \in H(\mathbf{C})$. By 1.4.3 and 1.4.7 in [15] we know from (11) that, for $p>0$ and $k \geq 0$,

$$
\begin{aligned}
& \int_{\mathbf{C}^{n}}\left|f(z) \omega^{k}(z) e^{-t|z|^{2}}\right|^{p} d A(z) \\
= & \frac{1}{2 \pi} \int_{0}^{\infty} r^{2 n-1} d r \int_{S} d \sigma(\zeta) \frac{1}{2 \pi} \int_{-\pi}^{\pi}\left|f_{\zeta}\left(r e^{i \theta}\right) \omega^{k}(r) e^{-t r^{2}}\right|^{p} d \theta \\
= & \int_{S} d \sigma(\zeta) \int_{\mathbf{C}}\left|f_{\zeta}(u) \omega^{k}(u) e^{-t|u|^{2}}\right|^{p}|u|^{2(n-1)} d A(u) \\
\leq & C \int_{S} d \sigma(\zeta) \int_{\mathbf{C}}\left|\left(f_{\zeta}\right)^{\prime}(u) \omega^{k+1}(u) e^{-t|u|^{2}}\right|^{p}|u|^{2(n-1)} d A(u) .
\end{aligned}
$$

Since $\frac{\Re f(u \xi)}{u}$ is holomorphic in $u \in \mathbf{C}$, we get

$$
\begin{aligned}
& \int_{\{z \in \mathbf{C}:|z|<1\}}\left|\frac{\Re f(u \zeta)}{u} \omega^{k+1}(u) e^{-t|u|^{2}}\right|^{p}|u|^{2(n-1)} d A(u) \\
\leq & C \frac{1}{2 \pi} \int_{-\pi}^{\pi}\left|\frac{\Re f\left(e^{i \theta} \zeta\right)}{e^{i \theta}}\right|^{p} d \theta \\
\leq & C \int_{\{z \in \mathbf{C}: 1<|z|<2\}}\left|\Re f(u \zeta) \omega^{k+2}(u) e^{-t|u|^{2}}\right|^{p}|u|^{2(n-1)} d A(u) .
\end{aligned}
$$

Then

$$
\begin{aligned}
& \int_{\mathbf{C}}\left|\frac{\Re f(u \zeta)}{u} \omega^{k+1}(u) e^{-t|u|^{2}}\right|^{p}|u|^{2(n-1)} d A(u) \\
\leq & C \int_{\mathbf{C}}\left|\Re f(u \zeta) \omega^{k+2}(u) e^{-t|u|^{2}}\right|^{p}|u|^{2(n-1)} d A(u) .
\end{aligned}
$$

Apply Proposition 6.4.4 in [15] to obtain

$$
\begin{aligned}
& \int_{\mathbf{C}^{n}}\left|f(z) \omega^{k}(z) e^{-t|z|^{2}}\right|^{p} d A(z) \\
\leq & C \int_{S} d \sigma(\zeta) \int_{\mathbf{C}}\left|\frac{\Re f(u \zeta)}{u} \omega^{k+1}(u) e^{-t|u|^{2}}\right|^{p}|u|^{2(n-1)} d A(u) \\
\leq & C \int_{S} d \sigma(\zeta) \int_{\mathbf{C}}\left|\Re f(u \zeta) \omega^{k+2}(u) e^{-t|u|^{2}}\right|^{p}|u|^{2(n-1)} d A(u) \\
\leq & C \int_{\mathbf{C}^{n}}\left|\Re f(z) \omega^{k+2}(z)\right|^{p} e^{-t|z|^{2}} d A(z),
\end{aligned}
$$


provided $f(0)=0$. Take $k=0,2,4, \cdots, 2(m-1)$ inductively in the above estimate (15) and notice that $\Re^{k} f(0)=0$ for $k \geq 1$ :

$$
\begin{aligned}
& \int_{\mathbf{C}^{n}}\left|f(z) e^{-t|z|^{2}}\right|^{p} d A(z) \\
\leq & C\left\{\int_{\mathbf{C}^{n}}\left|[f(z)-f(0)] e^{-t|z|^{2}}\right|^{p} d A(z)+|f(0)|^{p}\right\} \\
\leq & C\left\{\int_{\mathbf{C}^{n}}\left|\Re f(z) \omega^{2}(z) e^{-t|z|^{2}}\right|^{p} d A(z)+|f(0)|^{p}\right\} \\
& \ldots \ldots \\
\leq & C\left\{\int_{\mathbf{C}^{n}}\left|\Re^{m} f(z) \omega^{2 m}(z) e^{-t|z|^{2}}\right|^{p} d A(z)+|f(0)|^{p}\right\} .
\end{aligned}
$$

This gives the desired estimate $(\mathrm{A}) \leq C(\mathrm{C})$.

To prove $(\mathrm{A}) \leq C(\mathrm{~B})$, we notice that $|\Re f(z)| \leq|z||\nabla f(z)| \leq C|z| \sum_{j=1}^{n}\left|\frac{\partial f}{\partial z_{j}}\right|$. By (14) we have

$$
\int_{\mathbf{C}^{n}}\left|f(z) \omega^{k}(z) e^{-t|z|^{2}}\right|^{p} d A(z) \leq C\left\{|f(0)|^{p}+\int_{\mathbf{C}^{n}} \sum_{j=1}^{n}\left|\frac{\partial f}{\partial z_{j}} \omega^{k+1}(z) e^{-t|z|^{2}}\right|^{p} d A(z)\right\} .
$$

Inductively,

$$
\begin{aligned}
& \int_{\mathbf{C}^{n}}\left|f(z) \omega^{k}(z) e^{-t|z|^{2}}\right|^{p} d A(z) \\
\leq & C\left\{\sum_{|\alpha|=m} \int_{\mathbf{C}^{n}}\left|\partial^{\alpha} f \omega^{k+m}(z) e^{-t|z|^{2}}\right|^{p} d A(z)+\sum_{|\alpha| \leq m-1}\left|\partial^{\alpha} f(0)\right|^{p}\right\} .
\end{aligned}
$$

Taking $k=0$ in the above inequality we see that $(\mathrm{A}) \leq C(\mathrm{~B})$.

We now prove that $(\mathrm{C})$ can be dominated by (B). An elementary calculation shows that there are some constants $a_{\alpha}$ such that

$$
\Re^{m} f(z)=\sum_{|\alpha| \leq m} a_{\alpha} z^{\alpha} \partial^{\alpha} f(z) \text { for } f \in H\left(\mathbf{C}^{n}\right) .
$$

Then

$$
\left|\Re^{m} f(z) \omega^{2 m}(z)\right|^{p} \leq C \sum_{|\alpha| \leq m}\left|\partial^{\alpha} f(z) \omega^{m}(z)\right|^{p} \leq C \sum_{|\alpha| \leq m}\left|\partial^{\alpha} f(z) \omega^{|\alpha|}(z)\right|^{p} .
$$

It yields

$$
\begin{aligned}
& \int_{\mathbf{C}^{n}}\left|\Re^{m} f(z) \omega^{2 m}(z) e^{-t|z|^{2}}\right|^{p} d A(z) \\
\leq & C \sum_{|\alpha| \leq m} \int_{\mathbf{C}^{n}}\left|\partial^{\alpha} f(z) \omega^{|\alpha|}(z) e^{-t|z|^{2}}\right|^{p} d A(z) \\
\leq & C \sum_{|\alpha|=m}\left\{\sum_{|\beta| \leq m-|\alpha|-1}\left|\partial^{\beta+\alpha} f(0)\right|^{p}+\int_{\mathbf{C}}\left|\partial^{\alpha} f(z) \omega^{m}(z) e^{-t|z|^{2}}\right|^{p}\right\} \\
= & C\left\{\sum_{|\alpha| \leq m-1}\left|\partial^{\alpha} f(0)\right|^{p}+\sum_{|\alpha|=m} \int_{\mathbf{C}}\left|\partial^{\alpha} f(z) \omega^{m}(z) e^{-t|z|^{2}}\right|^{p}\right\} .
\end{aligned}
$$


Therefore, (C) can be dominated by (B). The proof of the theorem is completed.

Remark. In [7, it is claimed that "in general, the Fock space cannot be characterized in terms of membership of partial derivatives in $L^{p}$ spaces" (see pages 470 and 483 therein). Our Theorem 2.1 shows that the claim in [7] is false.

\section{The extended Cesaro operator}

In this section we are going to give some application of the estimate in the previous section. For this purpose, we need Fock Carleson measures which appear in 9 .

Definition. Let $0<p, q<\infty$ and let $\mu$ be a positive Borel measure on $\mathbf{C}^{n}$ (denoted by $\mu \geq 0)$. We call $\mu$ a $(p, q)$-Fock Carleson measure if the embedding operator $i: F_{\alpha}^{p} \rightarrow L_{\alpha}^{q}(d \mu)$ is bounded; i.e. there exists some constant $C$ such that for all $f \in F_{\alpha}^{p}$,

$$
\|f\|_{L_{\alpha}^{q}(d \mu)}=\left(\int_{\mathbf{C}^{n}}\left|f(z) e^{-\frac{\alpha}{2}|z|^{2}}\right|^{q} d \mu(z)\right)^{\frac{1}{q}} \leq C\|f\|_{p, \alpha} .
$$

We will use $\|\mu\|$ to stand for the operator norm of $i$ from $F_{\alpha}^{p}$ to $L_{\alpha}^{q}(d \mu)$. Also, we call $\mu$ a vanishing $(p, q)$-Fock Carleson measure if

$$
\lim _{j \rightarrow \infty} \int_{\mathbf{C}^{n}}\left|f_{j}(z) e^{-\frac{\alpha}{2}|z|^{2}}\right|^{q} d \mu(z)=0
$$

whenever $\left\{f_{j}\right\}$ is a bounded sequence in $F_{\alpha}^{p}$ that converges to 0 uniformly on compact subsets of $\mathbf{C}^{n}$ as $j \rightarrow \infty$.

The following three lemmas (Lemma 3.1, 3.2 and 3.3) are from [9] as well.

Lemma 3.1. Let $0<p \leq q<\infty$, and let $\mu \geq 0$. Then the following statements are equivalent:

(1) $\mu$ is a $(p, q)$-Fock Carleson measure;

(2) $\mu(B(\cdot, \delta))$ is bounded on $\mathbf{C}^{n}$ for some (or any) $\delta>0$.

Furthermore,

$$
\|\mu\| \simeq \sup _{z \in \mathbf{C}^{n}} \mu(B(z, \delta))^{\frac{1}{q}} .
$$

Lemma 3.2. Let $0<p \leq q<\infty$, and let $\mu \geq 0$. Then the following statements are equivalent:

(1) $\mu$ is a vanishing $(p, q)$-Fock Carleson measure;

(2) $\mu(B(z, \delta)) \rightarrow 0$ as $z \rightarrow \infty$ for some (or any) $\delta>0$.

Lemma 3.3. Let $0<q<p<\infty$, and let $\mu \geq 0$. Set $s=\frac{p}{q}$. Then the following statements are equivalent:

(1) $\mu$ is a $(p, q)$-Fock Carleson measure;

(2) $\mu$ is a vanishing $(p, q)$-Fock Carleson measure;

(3) $\mu(B(\cdot, \delta)) \in L^{s^{\prime}}(d A)$ for some (or any) $\delta>0$ with $s^{\prime}$ the conjugate exponent of $s$.

Furthermore,

$$
\|\mu\| \simeq\|\mu(B(\cdot, \delta))\|_{L^{s^{\prime}}(d A)}^{\frac{1}{q}} .
$$

The next lemma is a generalization of the well-known Liouville theorem in higher dimension. 
Lemma 3.4. Let $0<p \leq \infty$ and $m>0$. Then for $f \in H\left(\mathbf{C}^{n}\right)$, the limit $\lim _{r \rightarrow \infty} \frac{M_{p}(f, r)}{r^{m}}$ exists (or $\frac{\overline{M_{p}}(f, r)}{r^{m}}$ remains bounded on $[0, \infty)$ ) if and only if $f$ is a polynomial of degree not more than $m$.

Proof. If $f(z)=\sum_{|\alpha| \leq J} a_{\alpha} z^{\alpha}$ with $J \leq m$, it is easy to verify that

$$
\begin{aligned}
\lim _{r \rightarrow \infty} \frac{M_{p}(f, r)}{r^{m}} & =\lim _{r \rightarrow \infty}\left\{\int_{S}\left|\sum_{|\alpha| \leq J} a_{\alpha} \frac{1}{r^{J-|\alpha|}} \zeta^{\alpha}\right|^{p} d \sigma(\zeta)\right\}^{\frac{1}{p}} \frac{1}{r^{m-J}} \\
& =\left\{\int_{S}\left|\sum_{|\alpha|=J} a_{\alpha} \zeta^{\alpha}\right|^{\frac{1}{p}} d \sigma(\zeta)\right\}_{r \rightarrow \infty}^{p} \lim _{r \rightarrow m} \frac{1}{r^{m-J}} \\
& =\left\{\int_{S}\left|\sum_{|\alpha|=J} a_{\alpha} \zeta^{\alpha}\right|^{\frac{1}{p}} d \sigma(\zeta)\right\}^{p} \delta_{m, J},
\end{aligned}
$$

where $\delta_{m, J}=1$ if $m=J$ and $\delta_{m, J}=0$ if $m \neq J$. Conversely, if $\lim _{r \rightarrow \infty} \frac{M_{p}(f, r)}{r^{m}}$ exists, then we have some constant $C$ such that

$$
\frac{M_{p}(f, r)}{r^{m}} \leq C
$$

for all $r>1$. Write $f(z)=\sum_{|\alpha| \geq 0} b_{\alpha} z^{\alpha}$. Similar to the proof of the Liouville theorem in standard complex analysis, for any multi-index $\alpha$ with $|\alpha|>m$, with the Cauchy estimate and the subharmonicity of $|f|^{p}$,

$$
\left|b_{\alpha}\right| \leq C \frac{\max _{|\zeta|=r}|f(\zeta)|}{r^{|\alpha|}} \leq C \frac{M_{p}(f, 2 r)}{r^{m}} \frac{1}{r^{|\alpha|-m}} \rightarrow 0
$$

as $r \rightarrow \infty$. Therefore, $f$ is a polynomial of degree not more than $m$. The proof is completed.

Theorem 3.1. Let $0<p \leq q<\infty$, and let $g \in H\left(\mathbf{C}^{n}\right)$. Then $T_{g}$ is bounded from $F_{\gamma}^{p}$ to $F_{\gamma}^{q}$ if and only if $g$ is a polynomial of degree not more than two. Also, $T_{g}$ is compact from $F_{\gamma}^{p}$ to $F_{\gamma}^{q}$ if and only if $g$ is a polynomial of degree not more than one. Furthermore,

$$
\left\|T_{g}\right\| \simeq \sup _{z \in \mathbf{C}^{n}}\left|\Re g(z) \omega^{2}(z)\right| .
$$

Proof. Suppose $g \in H\left(\mathbf{C}^{n}\right)$; as in [8] we have $\Re T_{g} f(z)=f(z) \Re g(z)$. Then by Theorem 2.1,

$$
\left\|T_{g} f\right\|_{q, \gamma} \simeq\left\{\int_{\mathbf{C}^{n}}\left|f(z) e^{-\alpha|z|^{2}}\right|^{q}\left|\Re g(z) \omega^{2}(z)\right|^{q} d A(z)\right\}^{\frac{1}{q}}
$$

Therefore, the operator $T_{g}$ is bounded if and only if

$$
\left\{\int_{\mathbf{C}^{n}}\left|f(z) e^{-\alpha|z|^{2}}\right|^{q}\left|\Re g(z) \omega^{2}(z)\right|^{q} d A(z)\right\}^{\frac{1}{q}} \leq C\|f\|_{p, \gamma},
$$

equivalently, if and only if

$$
d \mu=\left|\Re g(z) \omega^{2}(z)\right|^{q} d A(z) \text { is a }(p, q) \text {-Fock Carleson measure. }
$$


By the subharmonicity of $|\Re g(z)|^{q}$, for $z \in \mathbf{C}^{n}$ there holds

$$
\begin{aligned}
\left|\Re g(z) \omega^{2}(z)\right|^{q} & \leq \omega^{2 q}(z) \frac{1}{|D(z, 1)|} \int_{D(z, 1)}|\Re g(z)|^{q} d A(z) \\
& \leq C \int_{D(z, 1)}\left|\Re g(z) \omega^{2}(z)\right|^{q} d A(z) .
\end{aligned}
$$

Lemma 3.1 tells us that $d \mu=\left|\Re g(z) \omega^{2}(z)\right|^{q} d A(z)$ is a $(p, q)$-Fock Carleson measure if and only if $\Re g(z) \omega^{2}(z)$ is bounded in $\mathbf{C}^{n}$. This and Lemma 3.4 imply that $T_{g}$ is bounded from $F_{\gamma}^{p}$ to $F_{\gamma}^{q}$ if and only if $\Re g(z)$ is a polynomial of degree not more than 2. In other words, $g$ is a polynomial of degree not more than 2 .

For the compactness of $T_{g}$, estimate (17) tells us that $T_{g}$ is compact from $F_{\gamma}^{p}$ to $F_{\gamma}^{q}$ if and only if $d \mu=\left|\Re g(z) \omega^{2}(z)\right|^{q} d A(z)$ is a vanishing $(p, q)$-Fock Carleson measure which is equivalent to the fact that $\lim _{z \rightarrow \infty} \Re g(z) \omega^{2}(z)=0$, as Lemma 3.2 implies. Lemma 3.4 tells us that $\Re g$ (and equivalently $g$ ) is a polynomial of degree not more than 1.

The operator norm estimate (16) comes from (18) and Lemma 3.1. The proof is completed.

Theorem 3.2. Let $0<q<p<\infty$, and let $g \in H\left(\mathbf{C}^{n}\right)$. Then the following statements are equivalent:

(A) $T_{g}$ is bounded from $F_{\gamma}^{p}$ to $F_{\gamma}^{q}$;

(B) $T_{g}$ is compact from $F_{\gamma}^{p}$ to $F_{\gamma}^{q}$;

(C) $g$ is a polynomial of degree not more than 1 if $\frac{p q}{p-q}>2 n$ and $g$ is constant if $\frac{p q}{p-q} \leq 2 n$.

Furthermore,

$$
\left\|T_{g}\right\| \simeq\left\{\int_{\mathbf{C}^{n}}\left|\Re g(z) \omega^{2}(z)\right|^{\frac{p q}{p-q}} d A(z)\right\}^{\frac{1}{q}} .
$$

Proof. Lemma 3.3 and (17) imply that (A) and (B) are equivalent, and each of them is equivalent to

$$
\int_{\mathbf{C}^{n}}\left|\Re g(z) \omega^{2}(z)\right|^{\frac{p q}{p-q}} d A(z)=\int_{0}^{\infty}\left\{\frac{M_{\frac{p q}{p-q}}(\Re g, r)}{(1+r)^{2}}\right\}^{\frac{p q}{p-q}} r^{2 n-1} d r<\infty .
$$

With the help of Lemma 3.4 we see that (20) is equivalent to the fact that $g$ is a polynomial of degree not more than 1 if $\frac{p q}{p-q}>2 n$ or that $g$ is constant if $\frac{p q}{p-q} \leq 2 n$. Estimate (19) comes from Lemma 3.3 and (18). The proof is completed.

\section{ACKNOWLEDGEMENT}

The author expresses his thanks to the referee for a careful reading of the manuscript.

\section{REFERENCES}

[1] A. Aleman, J. Cima, An integral operator on $H^{p}$ and Hardy's inequality, J. Anal. Math., 85(2001), 157-176. MR 1869606 (2002k:30068)

[2] A. Aleman, A. G. Siskakis, An integral operator on $H^{p}$, Complex Variables, 28(1995), 149158. MR.1700079 (2000d:47050)

[3] A. Aleman, A. G. Siskakis, Integration operators on Bergman spaces, Indiana University Math. J., 46(1997), 337-356. MR.1481594(99b:47039) 
[4] C. A. Berger, L. A. Coburn, Toeplitz operators on the Segal-Bargmann space, Trans. Amer. Math. Soc., 301(1987), 813-829. MR882716 (88c:47044)

[5] B. R. Choe, K. H. Izuchi, H. Koo, Linear sums of two composition operators on the Fock space, J. Math. Anal. Appl., 369(2010), 112-119. MR2643851 (2011c:47044)

[6] L. A. Coburn, J. Isralowitz, B. Li, Toeplitz operators with BMO symbols on the SegalBargmann space, Trans. Amer. Math. Soc., 363(2011), 3015-3030. MR2775796 (2012h:47049)

[7] O. Furdui, On a class of integral operators, Integr. Equ. Op. Theory, 60(2008), 469-483. MR2390439(2009a:47067)

[8] Z. J. Hu, Extended Cesàro operators on Bergman spaces, J. Math. Anal. Appl., 296(2004), 435-454. MR2075175 (2005e:47087)

[9] Z. J. Hu, X. F. Lv, Toeplitz operators from one Fock space to another, Integr. Equ. Op. Theory, 70(2011), 541-559. MR2819157

[10] Z. J. Hu, X. M. Tang, Schatten(-Herz) class extended Cesàro operators on Bergman spaces in the unit ball of $\mathbf{C}^{n}$, Proc. Amer. Math. Soc., 138(2010), 2803-2814. MR2644894 (2011d:47077)

[11] D. H. Luecking, Forward and reverse Carleson inequalities for functions in Bergman spaces and their derivatives, Amer. J. Math., 107(1985), 85-111. MR778090 (86g:30002)

[12] F. J. Martin-Reyes, E. Sawyer, Weighted inequalities for Riemann-Liouville fractional integrals of order one and greater, Proc. Amer. Math. Soc., 106(1989), 727-733. MR965246 (90a:26012)

[13] M. Pavlović, J. A. Peláez, An equivalence for weighted integrals of an analytic function and its derivative, Math. Nachr., 281(2008), 1612-1623. MR2462603 (2009m:30068)

[14] J. H. Shi, On the rate of growth of the means $M_{p}$ of holomorphic and pluriharmonic functions on bounded symmetric domains of $\mathbf{C}^{n}$, J. Math. Anal. Appl., 126(1987), 161-175. MR.900536 (89d:32011)

[15] W. Rudin, Function Theory in the Unit Ball of $\mathbf{C}^{n}$, Springer-Verlag, New York, 1980. MR601594(82i:32002)

[16] K. H. Zhu, The Bergman spaces, the Bloch space and Gleason's problem, Trans. Amer. Math. Soc., 309(1988), 253-265. MR931533 (89j:46025)

[17] K. H. Zhu, Operator Theory in Function Spaces, Mathematical Surveys and Monographs, 138, American Mathematical Society, 2007. MR2311536(2008i:47064)

Department of Mathematics, Huzhou Teachers College, Huzhou, Zhejiang, 313000 , People's Republic of China

E-mail address: huzj@hutc.zj.cn 